\title{
Predicting the Effect of Nano-Structural Parameters on the Elastic Properties of Carbon Nanotube-Polymeric based Composites
}

\author{
AHMAD ALMAGABLEH ${ }^{1}$, FARIS M. AL-OQLA ${ }^{1 *}$ and MOHAMMAD A. \\ OMARI $^{2}$ \\ ${ }^{1}$ Department of Mechanical Engineering, Faculty of Engineering, The Hashemite \\ University, Zarqa 13133, Jordan \\ ${ }^{2}$ Department of Mechanical Engineering, Jordan University of Science and Technology, \\ Irbid, Jordan
}

(Received on October 09, 2016, Revised on December 16, 2016)

\begin{abstract}
Discrepancy in reported elastic properties for nanocomposites is argued to be most likely a result of either variations in the size of reinforcement or lack of control of the composite microstructure. In general, there will be a size variation in nanotubes in a given composite, contribution from each nanotube diameter and the volume percentage that tubes of a definite diameter occupy within the composite toward the overall elastic modulus is modeled. In this work, Digimat-FE is used to generate a realistic three dimensional microstructure for the current carbon nanotube/ epoxy composite. A system of aligned carbon nanotubes embedded in epoxy matrix is modeled. In the system of aligned multi walled carbon nanotubes, the entire volume of the model has been divided into finite individual sub-composites, each one containing a specific nanotube diameter with a local volume fraction. A second model showed a single representative volume element for the current nano-composite, in which the carbon nanotubes were simulated as a randomly (fully) dispersed, where all particles have been separated from each other. Moreover, a micromechanical approach for modeling short fiber composites was developed to account for the structure of the multi-walled carbon nanotube reinforcement and predict the elastic modulus of the nanocomposite as a function of the constituent properties, reinforcement geometry and nanotube structure. Finite element results show increase in elastic modulus with increasing aspect ratio for composites with high filler loading (3 vol\%). Micromechanical predictions highlight the structure or size influence of the nanotube reinforcement on the properties of the nanocomposite. The nanocomposite elastic properties were found to particularly be sensitive to the nanotube diameter, since larger diameter nanotubes showed a lower effective modulus and occupied a greater volume fraction in the composite relative to smaller-diameter nanotubes.
\end{abstract}

Keywords: Carbon nanotubes; Nanocomposites; Representative volume element; Mechanical properties; Nano-fibers.

\section{Introduction}

Dramatic improvements in mechanical properties can be achieved by incorporation of a few weight percentages of layered particles in polymer matrices[1]. Layered silicates have a thickness of $1 \mathrm{~nm}$ and lateral dimensions of $30 \mathrm{~nm}$ to several microns are commonly used as reinforcement in polymeric based composites. The large aspect ratios of layered silicates are thought to be mainly responsible for the enhanced mechanical properties of 
particulate-polymer nanocomposites. On the contrary, the amazing large specific surface area of carbon nanotubes produces very big attractive forces (van der Waals forces) between the molecules, bringing a strong tendency to percolate. Their tangled yarn-like structure is an additional barrier to be overcome in order to achieve a uniform dispersion $[2,3]$.

The mechanical performance of particulate-polymer composites is far lower than the predicted values. In order to convey the excellent properties of filler into the composites and reach superior characteristics, filler need to be evenly dispersed in the matrix, which is one of the major preconditions for the utilization of reinforcements. Furthermore, mechanical properties of polymeric based composites depend strongly on the particle size, particle-matrix interface adhesion and particle loading. Particle size has an obvious effect on these mechanical properties. Sumita et al.[4] underlined the interest of replacing microscale silica by its nanoscale counterpart, since nanoscale silica particles possess superior mechanical properties. They found that these nanoparticles give higher rigidity and superior yield strength to the polymers. Similarly, alumina trihydrate filled epoxy containing smaller particles show higher fracture toughness [5]. Particle-matrix interface adhesion and particle loading are two important factors that also affect mechanical properties.

Epoxy resins on the other hand, are normally used in the formation of carbon nanotubes/ polymeric based-composites that can work as multifunctional materials with high stiffness, light weight and high electrical conductivity as well as other desired economic properties for many industrial applications [6-13]. Epoxy resins start in a molten form to assist dispersion of carbon nanotubes. Subsequently, the solution is cured after a hardener is added to form a hard-state composite. Several means are followed to disperse particles in epoxy resins for making such composites like solution mixing and blending [14]. Such methods cannot produce major improvements in carbon nanotubes /epoxy composite due to lack of controlled orientation and ineffective dispersion of filler content in the epoxy resin matrix. Aligned carbon array were successfully fabricated from which unbroken sheets can be directly drawn[15]. These unbroken and aligned carbon yarn can be piled together to form a carbon nanotube/polymer composites [16].

Moreover, there is a large discrepancy in the reported mechanical properties, but little insight is provided regarding the structure of nanocomposites. For instance, it was reported that enhancement in elastic stiffness was lower than expected when the nanotube is treated as a solid fiber [16-19]. Poor correlation between the actual properties and the predicted ones is often argued by mentioning that there is inadequate load transfer at the filler/matrix interface [20-25], overlooking tube structure at the nano-level. Thus, several attempts have worked on functionalizing the nanotube surface to provide better bonding between the nanotube and the polymer resin[26]. However, perfection in interfacial properties has a far greater effect on the composite's tensile strength and fracture mechanism rather than the elastic stiffness at low strain loading. For example, particlematrix adhesion was found to be responsible for the tensile strength of glass bead filled polystyrene composites [27]. 
Furthermore, it is clear that elastic properties of particulate-filled polymeric nanocomposites are affected by particle size and particle content. A finite element approach is adopted to investigate effect of several nano-structure parameters on properties of nanotube based composites. Information on the structure of the nanocomposite is required in order to simulate the elastic stiffness of a nano-tube based composite. Since there is a variation of nanotube diameters in a given specimen, contribution from each nanotube diameter and the volume fraction that tubes of a specific diameter occupy within the composite toward the overall elastic modulus is modeled. A composite model of aligned multiwalled carbon nanotubes throughout epoxy matrix is simulated via Digimat-FE program. Next, a composite model of randomly dispersed nanotubes has been built keeping size distribution of filler same in both cases. Impact of diameter distribution of carbon nanotubes on the elastic properties is highlighted in both cases.

\section{Modeling of Carbon Nanotube Based Composites:}

In modeling the aligned multi-walled carbon nanocomposites, the whole volume has been divided into a finite number of individual sub-composites (layer) or representative volume element, each layer has inclusions of identified nanotube diameter and specific aspect ratio to reflect size distribution of filler that is experimentally obtained from TEM measurements. The nanotubes are simulated as aligned, dispersed, embedded in epoxy matrix. Impact of diameter distribution on the elastic properties is predicted. Volume fraction, $v_{n t}$, that tubes of a given diameter occupy within a composite can be calculated as follows:

$$
v_{n t}=\frac{\int_{\mathrm{d}_{\mathrm{n}}}^{\mathrm{d}_{\mathrm{n}}+\Delta d} v_{f} \cdot \psi(d) \mathrm{d}(d)}{v_{\mathrm{n}}}
$$

Where $\psi(d)$ is the size distribution function of nanotubes, normally obtained from experimental testing. $v_{f}$ is the volume fraction of tubes throughout the entire composite and the boundaries of integral are the range of diameters contained in the $n$th layer. $\mathrm{v}_{\mathrm{n}}$ is the fractional volume of the sub-composite (layer) which is $1 / n, n$ is the number of suggested layers. Specifications of carbon nanotubes used in each individual composite model are shown in Table 1.

Table 1: Specifications of the Five Layers-Composite Model, Case of 1 vol. \% Loading

\begin{tabular}{|c|c|c|c|}
\hline Layer & $\begin{array}{c}\text { Average diameter of nanotube } \\
(\mathbf{n m})\end{array}$ & $\begin{array}{c}\text { Local volume } \\
\text { fraction, } \mathbf{v}_{\mathbf{n t}}\end{array}$ & Aspect ratio \\
\hline 1 & 15 & 0.01 & 67 \\
\hline 2 & 25 & 0.018 & 40 \\
\hline 3 & 35 & 0.013 & 29 \\
\hline 4 & 45 & 0.005 & 22 \\
\hline 5 & 55 & 0.003 & 18 \\
\hline
\end{tabular}




\subsection{Digimat Finite Element Analysis}

Digimat-FE is software that applies a direct, realistic finite element (FE) illustration of a representative volume element for a composite microstructure. It stands on direct nonlinear finite element analysis (FEA) of a microstructure, presents precise predictions at the microscopic and macroscopic scales. Moreover, it suggests a large number of parameters and factors to design a microstructure of choice. One of the choices is to implement agglomerated inclusions (clustering). The client can state the desired quantity of clusters, and the level of clustering. Further, microstructure can have spherical reinforcements, made of various sizes (size ratio 1:30). Moreover, distribution of filler dimensions can be identified in the phase window in Digimat-FE: a filler size can be either fixed, or arbitrary between a minimum and maximum value, or determined by a user defined size function as it is the case in our study.

A carbon nanotube is visualized as a graphene layer, where carbon atoms build a planar hexagonal structure, extruded into a seamless cylinder. Bonding between concentric layers is covalent, interactions between adjacent tubes (van der Waals forces) can be simulated by a coating surrounding each inclusion phase, with an elastic stiffness that emulates that interaction. This coating is then homogenized with the inclusion material, and the resultant effective particle is used for further homogenizing with voids to form an effective composite structure. The pretty poor interaction between the walls of the nanotubes results in nominal load transfer between those concentric layers of the nanotube. As a result of this weak bonding between the layers, the external layer of the multi-walled tube will take roughly the full load transferred at the nanotube/matrix interface. Thus, the outer wall of the nanotube is considered to behave as an effective solid fiber.

A finite element approach via Digimat software is conducted to simulate the elastic properties of the nanotube-based composite, at which the structure of the carbon nanotubes is built at nanoscale. Since this type of modeling is applied over a low strain loading, it can be assumed that interfacial stresses do not result in debonding of the reinforcement/matrix interface; therefore a perfect bonding is considered. Figure 1 shows a typical representative volume element geometry in Digimat for epoxy matrix filled with uniformly dispersed $1 \mathrm{wt}$. \% carbon nanotube. 


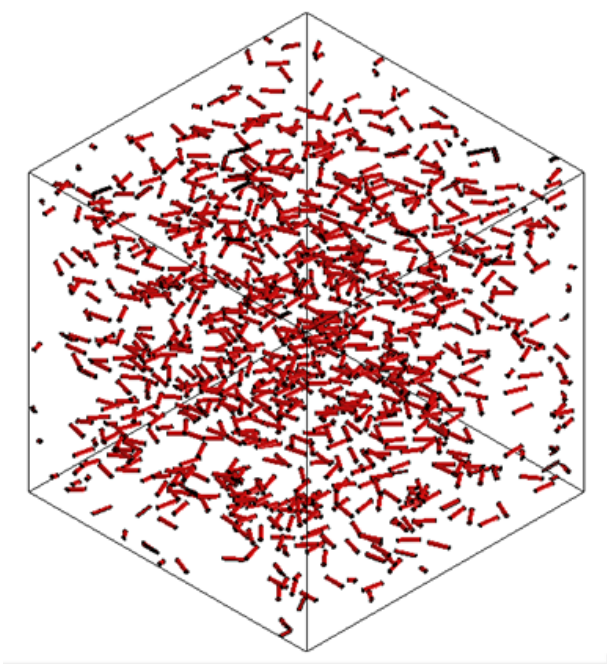

Figure 1: Representative Volume Model for Randomly Dispersed Carbon Nanotube in Epoxy

\subsection{Micromechanics}

Elastic stiffness of a short fiber-polymeric composite is generally determined by the elastic properties of its constituent (particle and matrix), particle loading and aspect ratio. Both nanotube and the effective fiber will undergo iso-strain condition when subjected to an external load. As the external force is same for both, stiffness of the effective fiber which is a solid cylinder can be expressed as a function of that for the nanotube, as given:

$$
E_{\text {eff }}=4 \frac{t}{d} E_{N T}
$$

Where the symbols NT and eff, refer to the nanotube and effective fiber, respectively. $t$, is the nanotube wall thickness and $(d)$, is the nanotube diameter. Halpin-Tsai theory is commonly used to evaluate modulus property for nano based composites [21], given as:

$$
E_{c}=E_{m} \frac{1+\xi \eta v_{f}}{1-\eta v_{f}}
$$

Where, $E_{c}, E_{f}, E_{m}$ and $v_{f}$, are composite stiffness, fiber stiffness, matrix stiffness and volume fraction of reinforcement, respectively. $\eta$, is given as:

$$
\eta=\frac{\left(E_{f} / E_{m}\right)-1}{\left(E_{f} / E_{m}\right)+\xi}
$$

$\zeta$ is the aspect ratio of the inclusion. Substitute equations (2), (4) in equation (3), get: 


$$
\frac{E_{C}}{E_{m}}=\frac{1+\xi v_{n t}\left(\frac{\left(E_{n t} / E_{m}\right)-(d / 4 t)}{\left(E_{n t} / E_{m}\right)+(l / 2 t)}\right)}{1-v_{n t}\left(\frac{\left(E_{n t} / E_{m}\right)-(d / 4 t)}{\left(E_{n t} / E_{m}\right)+(l / 2 t)}\right)}
$$

Equation (5) states the diameter-dependence of elastic properties for carbon nanotube based-composite. However, with a distribution of nanotube size throughout the composite, equation (5) cannot be used directly to predict the overall modulus. Therefore, the contribution from each nanotube diameter and the volume fraction that tubes of a specific diameter occupy within the composite toward the overall elastic modulus should be taken into account. Since the nanotubes are homogeneously dispersed and parallel throughout the matrix, the elastic modulus of the composite can be considered as a summation of parallel composites over the range of nanotube diameters. Each of the $\mathrm{N}$ sub-composites will have its own elastic modulus that depends on the local volume fraction of nanotubes with respect to a given diameter. The overall longitudinal stiffness of the composite can be expressed as sum of moduli weighed by the partial volume of each $n$th segment, as given below:

$$
E_{c}=E_{1} v_{n t 1}+E_{2} v_{n t 2}+\ldots . . E_{n} v_{n t}
$$

Where $E_{l}$ or $E_{n}$ is the elastic modulus of the layer calculated from equation (5) at the respective diameter of nanotubes included in the $n$th layer. The local volume fraction, $v_{n t}$, in each composite with specific nanotube diameter is calculated using Equation 1, as listed in Table 1.

\section{Results and Discussion}

Elastic properties of nanotube-reinforced composites depend strongly on particle size or aspect ratio, and particle loading. By knowing structures of both nanotube and nanocomposite, Digimat FEA can be used to build a model of nanotube-based composite and calculate the elastic modulus. A Digimat model for aligned filler composite is made of five individual composites; each one has its own volume fraction of nanotubes with respect to a given diameter in that layer. Besides, influence of randomly dispersed nanotubes on the tensile modulus is investigated. A single geometrical model for the current nano-composite is generated, in which reinforcements are simulated as a randomly (fully) dispersed, means that all particles have been separated from each other, keeping the size distribution function and filler content similar to the aligned composite system. Distribution of nanotube-diameter used, is given as per [16].

$$
\xi(d)=0.0234 e^{\left(-((d-31.8) / 5.8)^{2}\right)}+0.076 e^{\left(-((d-18) / 5.1)^{2}\right)}
$$

In order to simulate diameter distribution of nanotubes, size distributions of nanotubes, $\varphi$, is interpreted in the form of Gaussian probability function. The correlated Gaussian function shows a mean of $\left(27.5 \mathrm{~nm}^{3}\right)$ and a variance $(10.7)$ as seen in Figure 2. After generation the microstructure, the model for the aligned system is subjected to a quasi-static load in the fiber direction. The elastic stiffness results have been postprocessed from Digimat and presented for both cases: aligned and random distribution of filler. Alternatively, micromechanics theory for short fiber composites has been applied to 
predict the elastic modulus of aligned composite system as a function of constituent properties and nanotube structure.

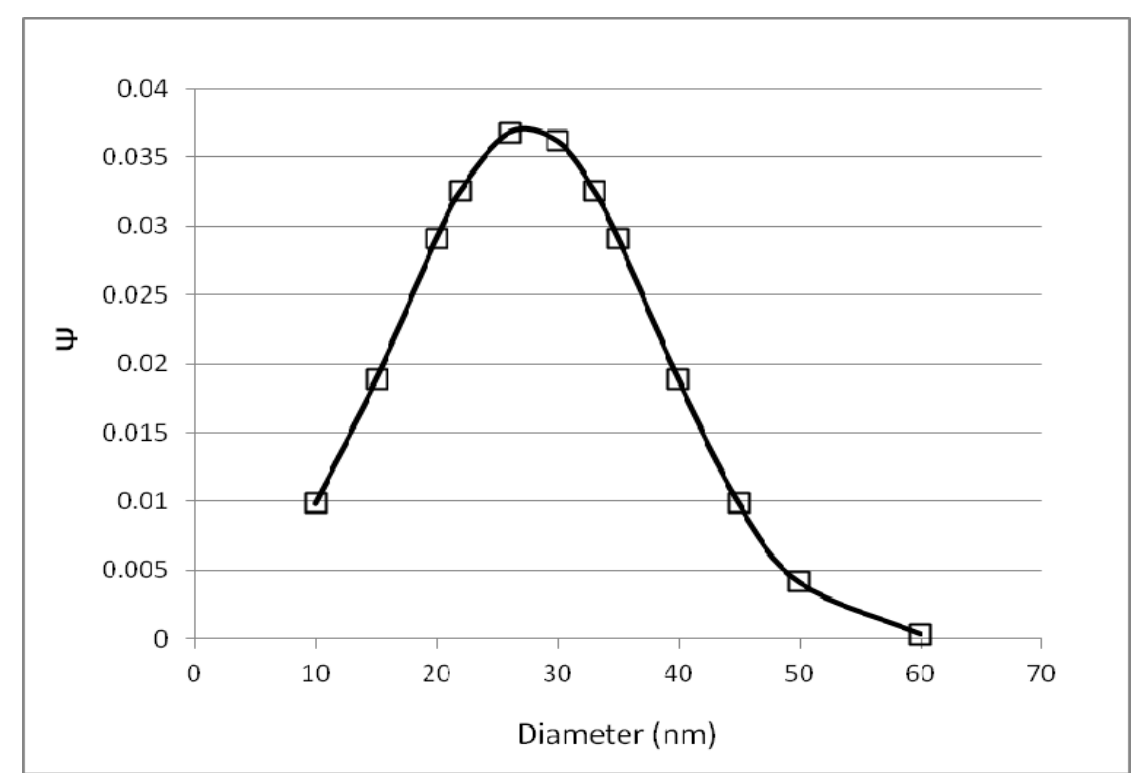

Figure 2: Probability Distribution (Gaussian) Function of Carbon Nanotubes

\subsection{FEA Results}

Digimat-FE has been used to create a realistic three dimensional microstructure of the current composite, it was exported in step formats, or failure indicator, for a given phase in the composite. For modeling nanotube based composite, two cases have been considered. First, a system of aligned tube-composite contains five individual layers or sub-composites, each one has its own volume fraction and aspect ratio of nanotubes as per Table 1. On the other hand, Figure 3 shows geometries for the aligned composite systems with two different aspect ratio of $3 . \%$ filler loading.

Figure 4 shows influence of volume fraction and aspect ratio (particle size) on the elastic modulus for aligned particles-composite. It can be shown that in these composites, the tubes were unbroken and aligned along the loading direction. When the composites were loaded in the direction of the filler orientation, the overall elastic modulus was shown to be increased as a result of load transfer to the nanotube reinforcements rather than polymer chain. 


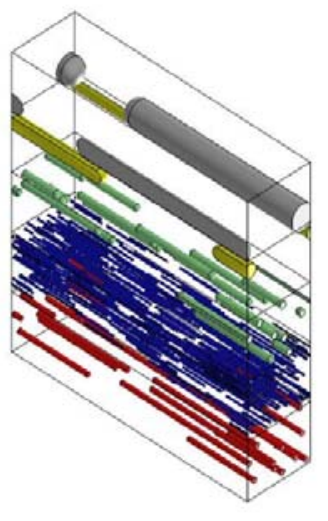

(a)

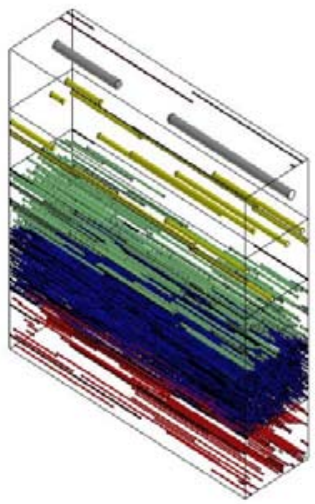

(b)

Figure 3: Digimat Finite Element Model of 3.\% Aligned Carbon Naotubes/ Epoxy Nanocomposite, a) Low Aspect Ratio and, b) High Aspect Ratio

Generally, as the filler content increases, the Young's modulus increases for a given aspect ratio. For composites of low volume fractions of carbon nanotubes (1-2 vol.\%), the modulus is almost insensitive to aspect ratio or particle size. However, composites with high nanotube content ( $3 \mathrm{vol} \%$ ), a remarkable increase in modulus was observed with increasing aspect ratio up to aspect ratio of 55 , (18 nm tubes-diameter and less), then followed by slight decrease in modulus with increasing tube-size. FE results imply that influence of reinforcement loading on elastic modulus is more pronounced than effect of aspect ratio, the later had shown little effect on composite stiffness.

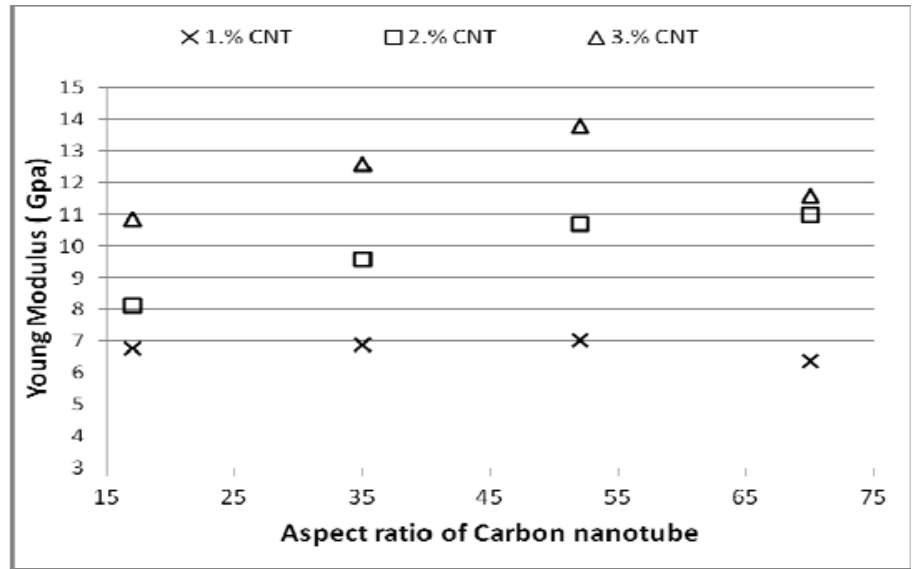

Figure 4: Influence of Nanotube Aspect Ratio, Volume Fraction on the Elastic Properties of Epoxy/ Aligned Composite System, Simulated via Finite Element Model 
Figure 5 presents periodic nanostructures, also referred to as representative volume element, that have been generated using Digimat -FE for the randomly placed inclusions. Figure 5 demonstrates microstructures for two volume fractions of inclusion phase, namely 0.005 and 0.01 filler loading. The diameter of nanotubes was varied according to the size distribution function as per Figure 2. Although the large-diameter nanotubes were relatively a small percentage of the total number of nanotubes, they occupied a significant percentage of volume within the composite (Figure 5-b). Clustering phenomena has been observed at the local regions, where inclusions with large size (diameter) are concentrated around a single distinct clustering plane. Due to the proximity of the inclusions around the clustering centers, stress concentrations appear. One clearly observes the higher stress levels $(3,500 \mathrm{MPa})$ in the inclusion phase for the higher filler content of 0.01 loading (Figure 7) as compared to $250 \mathrm{MPa}$, the maximum tensile stresses observed for the freeclustered case of 0.005 vol. filler conditions. Such high stress concentrations that are not observed for randomly placed inclusions in the case of $0.005 \mathrm{vol} \%$, could lead to debonding during loading. At this low volume fraction of inclusions, clustering was not prominent and did not significantly alter the macroscopic mechanical properties of the material. Such a placement of nano-inclusions was thus preferably avoided by the material scientists when trying to increase the stiffness of a base material.

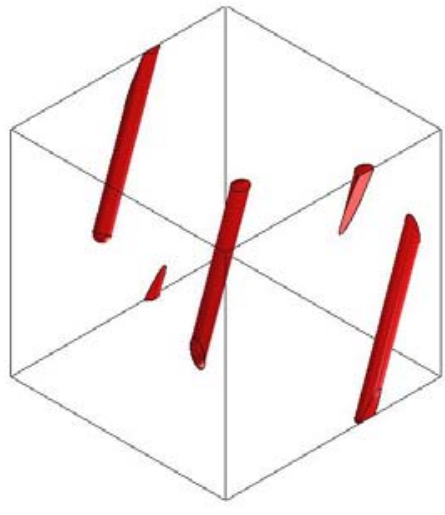

(a)

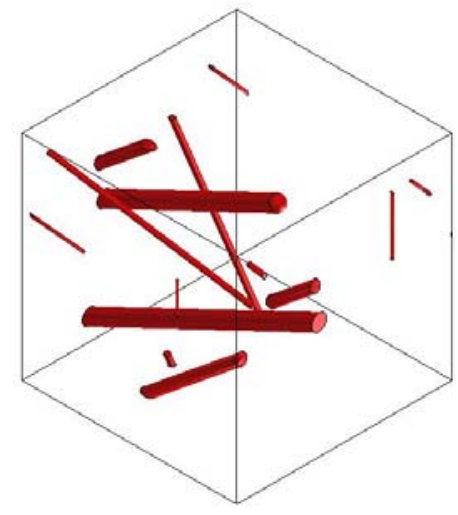

(b)

Figure 5: Three Dimensional Model of Randomly Dispersed Tubes ( $3 \mathrm{~nm} * 3 \mathrm{~nm} * 3 \mathrm{~nm})$, Generated by Digimat Following Gaussian Distribution of Nanotubes Diameter, a) 0.005 , and b) 0.01 vol.fraction

Figure 6 and Figure 7 in addition, illustrate the stress distribution in both matrix and inclusion phases for the randomly placed inclusions, following Gaussian size function, in the case of 0.005 and 0.01 filler loading, respectively. 

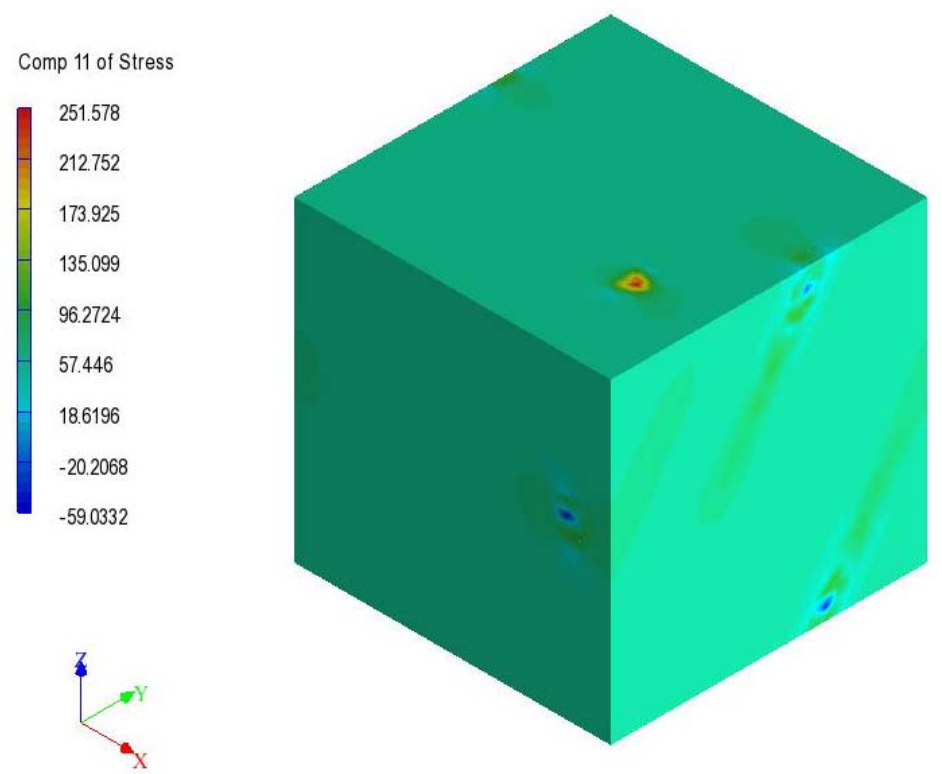

Figure 6: Stress Distribution for the Randomly Placed (free-clustered) Inclusions, case of 0.005 Filler Content.
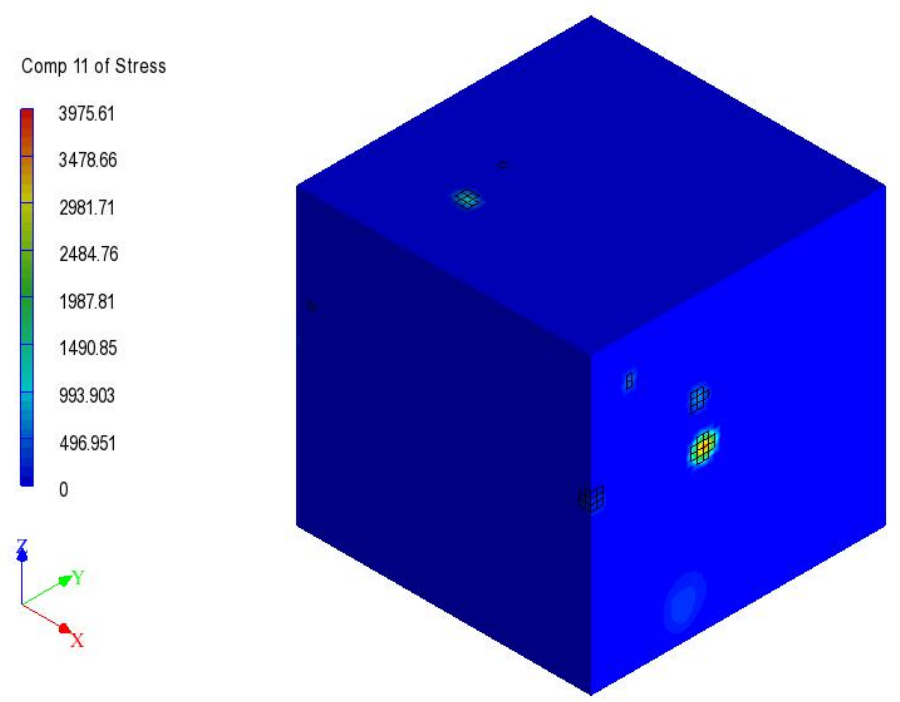

Figure 7: Stress Distribution for the Randomly Placed (clustered) Inclusions, Case of 0.01 Filler Content. 


\subsection{Micromechanics Results}

As an input into the micromechanical-Tsai-Hill model, the modulus of the nanotube, $E \mathrm{NT}$, was considered to be $1 \mathrm{TPa}$ and the modulus of the matrix, epoxy was $3.5 \mathrm{GPa}$. Note that microstructure along with the diameter distribution of the nanotube was considered as the added value to the common Tsai-hill theory. Figure 8 shows the influence of aspect ratio (nanotube diameter) and volume fraction on the composite elastic modulus for the aligned composite system, as predicted based on micromechanics. While there is a slight increase in elastic modulus with increasing volume fraction, keeping nanotube length constant, the size of the nanotubes (aspect ratio) plays the most significant role in the composite elastic modulus.

Based on micromechanics theory, composite modulus was found to be sensitive to filler aspect ratio or nanotube diameter. However, when the tube size is decreased below a threshold value of $35 \mathrm{~nm}$, there will be an obvious effect of particle size on the modulus predicted theoretically. This dependence of the composite elastic modulus on nanotube aspect ratio highlights the need to accurately model the distribution of nanotube diameters in the composite.

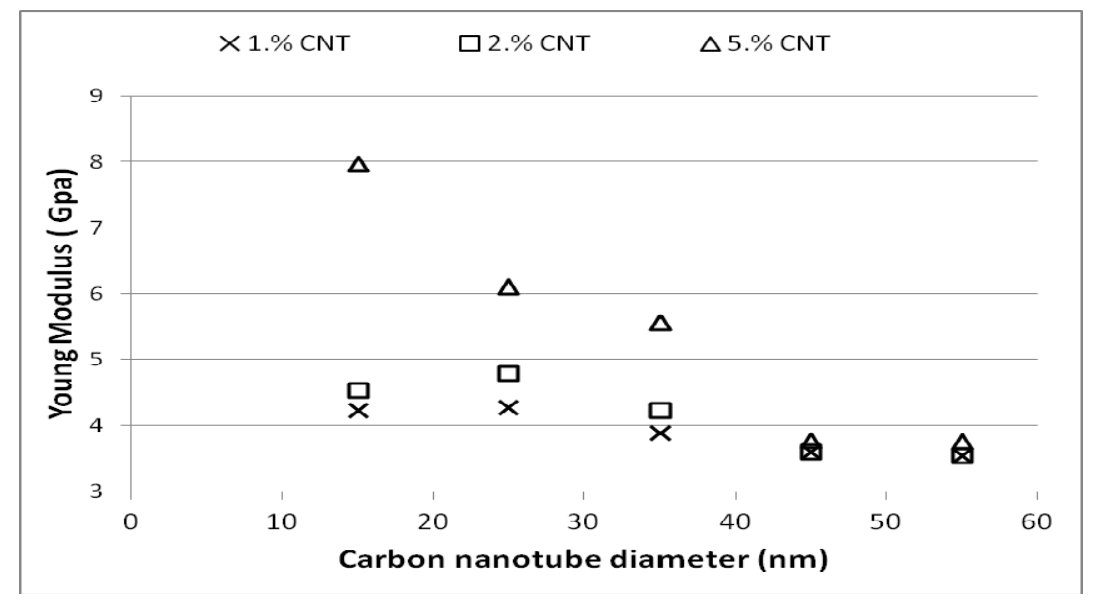

Figure 8: Influence of Nanotube Diameter, Volume Fraction on the Elastic Properties of an Aligned Nanocomposite System, Based on the Micromechanical Model

\section{Conclusions}

Digimat-FE has been used to generate a realistic three dimensional microstructure of carbon nanotubes/ epoxy composite. A system of aligned carbon nanotubes embedded in epoxy matrix was modeled in Digimat. In the system of aligned multiwalled carbon nanotubes, the entire volume of the model has been divided into finite individual composites, each layer containing a specific nanotube diameter with a local volume fraction. A second model showed a single layer for the current nano-composite, in which tubes were simulated as a randomly (fully) dispersed, where all particles have been separated from each other. The structure of the carbon nanotube was found to strongly influence the elastic properties of the nano-tube composite. Finite element results revealed 
that modulus is almost insensitive to aspect ratio or particle size for the aligned system under low filler content (1-2 vol\%). However, increase in elastic modulus was observed with increasing aspect ratio for those composites having high filler content (3 vol\%). Micromechanical predictions highlight structure or size influence of the nanotube reinforcement on the properties of the nanocomposite. The nanocomposite elastic properties were found to particularly be sensitive to the nanotube diameter (aspect ratio), since larger diameter nanotubes showed a lower effective modulus and occupied a greater volume fraction in the composite relative to smaller-diameter nanotubes.

\section{References}

[1]. Alexandre M., and P. Dubois. Polymer-Layered Silicate Nanocomposites: Preparation, Properties and Uses of a New Class of Materials. Materials Science and Engineering: R: Reports. 2000 Jun 15;28(1):1-63.

[2]. Yip M.C., Y.C. Lin, and C.L. Wu. Effect of Multi-Walled Carbon Nanotubes Addition On Mechanical Properties of Polymer Composites Laminate. Polymers \& Polymer Composites. 2011 Feb 10;19(2/3):131.

[3]. Sumfleth J., K. Prehn, M.H. Wichmann, S. Wedekind, and K. Schulte. A Comparative Study of the Electrical and Mechanical Properties of Epoxy Nanocomposites Reinforced by CVD-And Arc-Grown Multi-Wall Carbon Nanotubes. Composites Science and Technology. 2010 Jan 31;70(1):173-80.

[4]. Sumita M., T. Shizuma, K. Miyasaka, and K. Ishikawa. Effect of Reducible Properties of Temperature, Rate of Strain, And Filler Content On the Tensile Yield Stress of Nylon 6 Composites Filled with Ultrafine Particles. Journal of Macromolecular Science, Part B: Physics. 1983 Aug 1;22(4):601-18.

[5]. Radford K.C. The Mechanical Properties of an Epoxy Resin with A Second Phase Dispersion. Journal of Materials Science. 1971 Oct 1;6(10):1286-91.

[6]. AL-Oqla F.M., O.Y. Alothman, M. Jawaid, S.M. Sapuan, and M.H. Es-Saheb. Processing and Properties of Date Palm Fibers and its Composites. In Biomass and Bioenergy 2014 (pp. 1-25). Springer International Publishing.

[7]. Al-Oqla F.M., M.S. Sapuan, M.R. Ishak, and N.A. Aziz. Combined Multi-Criteria Evaluation Stage Technique as an Agro Waste Evaluation Indicator for Polymeric Composites: Date Palm Fibers as a Case Study. Bio Resources. 2014 Jun 17;9(3):460821.

[8]. Al-Oqla F.M., M.S. Salit, M.R. Ishak, and N.A. Aziz. Selecting Natural Fibers for BioBased Materials with Conflicting Criteria. American Journal of Applied Sciences. 2015 Jan 1;12(1):64.

[9]. Al-Oqla F.M., and S.M. Sapuan. Polymer Selection Approach for Commonly and Uncommonly Used Natural Fibers Under Uncertainty Environments. JOM. 2015 Oct 1;67(10):2450-63.

[10]. AL-Oqla F.M., S.M. Sapuan, M.R. Ishak, and A.A. Nuraini. A Model for Evaluating and Determining the Most Appropriate Polymer Matrix Type for Natural Fiber Composites. International Journal of Polymer Analysis and Characterization. 2015 Apr 3;20(3):191-205.

[11]. AL-Oqla F.M., and M.T. Hayajneh. A Design Decision-Making Support Model for Selecting Suitable Product Color to Increase Probability. In Design Challenge Conference: Managing Creativity, Innovation, And Entrepreneurship 2007.

[12]. AL-Oqla F.M., and A.A. Omar. An Expert-Based Model for Selecting the Most Suitable Substrate Material Type for Antenna Circuits. International Journal of Electronics. 2015 Jun 3;102(6):1044-55.

[13]. AL-Widyan M.I., and F.M. AL-Oqla. Utilization of supplementary Energy Sources for Cooling in Hot Arid Regions Via Decision-Making Model. International Journal of 
Engineering Research and Applications. 2011;1(4):1610-22.

[14]. Cheng Q.F., J.P. Wang, J.J. Wen, C.H. Liu, K.L. Jiang, Q.Q. Li, and S.S. Fan. Carbon Nanotube/Epoxy Composites Fabricated by Resin Transfer Molding. Carbon. 2010 Jan 31;48(1):260-6.

[15]. Liu K., Y. Sun, L. Chen, C. Feng, X. Feng, K. Jiang, Y. Zhao, and S. Fan. Controlled Growth of Super-Aligned Carbon Nanotube Arrays for Spinning Continuous Unidirectional Sheets with Tunable Physical Properties. Nano Letters. 2008 Feb $13 ; 8(2): 700-5$.

[16]. Thostenson E.T., and T.W. Chou. On The Elastic Properties of Carbon Nanotube-Based Composites: Modelling and Characterization. Journal of Physics D: Applied Physics. 2003 Feb 14;36(5):573.

[17]. Breton Y., G. Desarmot, J.P. Salvetat, S. Delpeux, C. Sinturel, F. Beguin, and S. Bonnamyc. Mechanical Properties of Multiwall Carbon Nanotubes/Epoxy Composites: Influence of Network Morphology. Carbon. 2004 Dec 31;42(5):1027-30.

[18]. AL-Oqla F.M., and M.A. Omari. Sustainable Biocomposites: Challenges, Potential and Barriers for Development. In Green Biocomposites 2017 (pp. 13-29). Springer International Publishing.

[19]. Sapuan S. M., W.H. Haniffah, and M. Faris. Effects of Reinforcing Elements on the Performance of Laser Transmission Welding Process in Polymer Composites: A Systematic Review. International Journal of Performability Engineering. 2016 Nov; 12(6):553.

[20]. Al-Oqla F.M., S.M. Sapuan, M.R. Ishak, and A.A. Nuraini. A Decision-Making Model for Selecting the Most Appropriate Natural Fiber-Polypropylene-Based Composites for Automotive Applications. Journal of Composite Materials. 2016 Feb 1;50(4):543-56.

[21]. Al-Oqla F.M., S.M. Sapuan, M.R. Ishak, and A.A. Nuraini. A Novel Evaluation Tool for Enhancing the Selection of Natural Fibers for Polymeric Composites Based On Fiber Moisture Content Criterion. BioResources. 2014 Nov 18;10(1):299-312.

[22]. Al-Oqla F.M., and S.M. Sapuan. Natural Fiber Reinforced Polymer Composites in Industrial Applications: Feasibility of Date Palm Fibers for Sustainable Automotive Industry. Journal of Cleaner Production. 2014 Mar 1;66:347-54.

[23]. Al-Oqla F.M., S.M. Sapuan, T. Anwer, M. Jawaid, and M.E. Hoque. Natural Fiber Reinforced Conductive Polymer Composites as Functional Materials: A Review. Synthetic Metals. 2015 Aug 31;206:42-54.

[24]. AL-Oqla F.M., S.M. Sapuan, and M. Jawaid. Integrated Mechanical-EconomicEnvironmental Quality of Performance for Natural Fibers for Polymeric-Based Composite Materials. Journal of Natural Fibers. 2016 Nov 1;13(6):651-9.

[25]. Sapuan S.M., F.L. Pua, Y.A. El-Shekeil, and F.M. Al-Oqla. Mechanical Properties of Soil Buried Kenaf Fibre Reinforced Thermoplastic Polyurethane Composites. Materials \& Design. 2013 Sep 30;50:467-70.

[26]. Brown M., and K. Jagannadham. Interfacial Effects in The Electrical Conductivity and Viscous Deformation of Multiwall Carbon Nanotube-Epoxy Composites Prepared By Sonication. Journal of Composite Materials. 2013 Dec 1;47(27):3413-20.

[27]. Dekkers M.E., and D. Heikens. The Effect of Interfacial Adhesion On the Tensile Behavior of Polystyrene-Glass - Bead Composites. Journal of Applied Polymer Science. 1983 Dec 1;28(12):3809-15.

Ahmad Almagableh is currently assistant Professor in Mechanical Engineering at the Hashemite University of Jordan. He has B.S an M.S. in Mechanical Engineering from Jordan University of Science and Technology at Jordan; he joined the nano-research group at the University of Mississippi in 2007 and earned Ph.D.in Engineering Mechanics from the Universityof Mississippi/ USA in 2011. His research interest is focused on 
experimental characterization, and modeling of mechanical behavior of nano-reinforced composites .

Faris M. AL-Oqla is currently an Assistant Professor of Mechanical Engineering at the Hashemite University (HU) / Jordan. He has B.Sc and M.Sc. in Mechanical Engineering from Jordan University of Science and Technology at Jordan. He joined the bio-composite technology research group at Universiti Putra Malaysia (UPM) in 2012 and he was awarded the International Graduate Research Fellowship (IGRF) / Department of Mechanical and Manufacturing Engineering (UPM) where he earned his Ph.D. in Mechanical Engineering in 2015. Dr. AL-Oqla has wide experience in both academic and industry as he was working as a lecturer in the college of engineering at King Faisal University/ KSA, Jordan University of Science and Technology and in the college of fine arts at Yarmouk University / Jordan. His research interests are natural fiber composites, materials selection for composites, green composites and decision making selection models. During his professional career he has several international publications, books, chapters in books and refereed conferences regarding mechanical engineering field particularly the polymeric- based composites.

Mohammad A. Omari is currently an assistant Professor in Mechanical Engineering at Jordan University of Science and technology/ Jordan. He earned Ph.D.in Engineering Mechanics from the USA in 2014. His research interest is focused on materials and their mechanical behavior and characterization. 